Pacific Journal of Mathematics

NONCOINCIDENCE INDEX OF MANIFOLDS 


\title{
NONCOINCIDENCE INDEX OF MANIFOLDS
}

\author{
Michael HofFman
}

\begin{abstract}
For a connected topological manifold $M$ we define the noncoincidence index of $M$, a topological invariant reflecting the abundance of fixed-point-free self-maps of $M$. We give some theorems on noncoincidence index and compute the noncoincidence index of the homogeneous manifold $U(n) / H$, where $H$ is conjugate to $U(1)^{k} \times U(n-k)$.
\end{abstract}

1. Introduction. Let $M$ be a manifold (connected locally Euclidean Hausdorff space). We define the noncoincidence index of $M$, \#M, as follows. If $M$ admits $k$ fixed-point-free self-maps, no pair of which has a coincidence, set $\# M \geq k+1$. If $\# M \geq i$ for all $i$, put $\# M=\infty$; otherwise, $\# M$ is the greatest number $i$ with $\# M \geq i$. (This definition is inspired by [5].)

Evidently a manifold has noncoincidence index 1 if and only if it has the fixed-point property. On the other hand, if a group $G$ acts freely on $M$, then $\# M \geq$ card $G$ if $G$ is finite and $\# M=\infty$ if $G$ is infinite. In particular, any connected nontrivial Lie group has noncoincidence index $\infty$.

As we see in $\$ 2$, many manifolds besides Lie groups have noncoincidence index $\infty$. In $\S 3$ we show how the Lefschetz coincidence theorem can be used to put a finite upper bound on $\# M$ for certain compact oriented manifolds $M$. These results are used in $\$ 4$ to compute the noncoincidence index of the homogeneous space $U(n) / H$ for $H$ conjugate to $U(1)^{k} \times$ $U(n-k)$. Section 5 is devoted to proving a classification theorem for endomorphisms of $H^{*}(U(n) / H ; \mathbf{Q})$ which is needed in $\S 4$.

I thank my colleague W. Homer for greatly improving Lemma 5.3, and I thank A. Dold for some helpful observations.

2. Sufficient conditions for $\# M=\infty$. In this section we give some sufficient conditions for a manifold $M$ to have $\# M=\infty$. The following result gives some easily checked homological conditions.

THEOREM 2.1. Let $M$ be a compact manifold. Then $\# M=\infty$ if either of the following is true:

1. $M$ has nonzero first Betti number, or

2. $\chi(M)=0$. 
Proof. For (1), see Corollary 5.1 of [5]. Now suppose $\chi(M)=0$. By [4], there is a map $s:[0,1] \times M \rightarrow M$ with $s(0, \cdot)=\mathrm{id}_{M}$ and $s(t, \cdot)$ : $M \rightarrow M$ fixed-point-free for $t>0$. Let $d$ be a metric for $M$, and set

$$
N(t)=\inf _{x \in M} d(s(t, x), x), \quad F(t)=\sup _{x \in M} d(s(t, x), x) .
$$

Then $F(t) \geq N(t)>0$ for $t>0$, and $F(t), N(t) \rightarrow 0$ as $t \rightarrow 0$. Choose $0<t_{k}<t_{k-1}<\cdots<t_{1} \leq 1$ so that $F\left(t_{l}\right)<N\left(t_{t-1}\right)$ : then

$$
x \rightarrow s\left(t_{i}, x\right), \quad 1 \leq i \leq k,
$$

is a set of $k$ fixed-point-free, noncoincident maps. Since we can do this for any $k, \# M=\infty$.

From the preceding result, we see that any odd-dimensional compact manifold has noncoincidence index $\infty$. It also follows that $\# M=\infty$ for any compact surface $M$, except $M=S^{2}$ and $M=\mathbf{R} P^{2}$ (of course \# $\mathbf{R} P^{2}$ $=1$, and we see in the next section that $\# S^{2}=2$ ).

The next result gives another useful sufficient condition for $\# M=\infty$.

THEOREM 2.2. Let $M$ be a compact manifold which admits a fixedpoint-free nonsurjective self-map. Then $\# M=\infty$.

Proof. Let $f: M \rightarrow M$ be fixed-point-free and nonsurjective. By Theorem 1.11 of [3], there is a path field nonsingular on the image of $f$, i.e. a map $s:[0,1] \times M \rightarrow M$ such that $s(0, \cdot)=\mathrm{id}_{M}$ and $s(t, \cdot)$ fixes no point of $f(M)$ for $t>0$. Let $d$ be a metric for $M$ and take $\varepsilon>0$ so that $d(f(x), x) \geq \varepsilon$ for $x \in M$. Then there is some $t_{0}>0$ so that

$$
\sup _{x \in M} d(s(t, x), x)<\varepsilon
$$

for $t<t_{0}$. Now proceed as in the proof of 2.1 ; set

$$
N(t)=\inf _{x \in f(M)} d(s(t, x), x), \quad F(t)=\sup _{x \in f(M)} d(s(t, x), x)
$$

(note $f(M)$ is compact) and choose $0<t_{k}<t_{k-1}<\cdots<t_{1}<t_{0}$ such that $F\left(t_{i}\right)<N\left(t_{i-1}\right)$. Then there are $k$ fixed-point-free noncoincident self-maps of $M$ given by

$$
x \rightarrow s\left(t_{l}, f(x)\right), \quad 1 \leq i \leq k .
$$

Since $k$ is arbitrary, $\# M=\infty$.

3. The Lefschetz coincidence theorem. In this section we show how the Lefschetz coincidence theorem can be used to put a finite upper bound on the noncoincidence index in some cases. As we see in the next 
section, such an upper bound combined with constructions of fixedpoint-free maps often gives the noncoincidence index exactly.

Throughout this section, $M$ will be a compact oriented $n$-manifold. We shall use the following version of the Lefschetz coincidence theorem: for a more general statement, see [9].

THEOREM 3.1. For maps $f, g: M \rightarrow M$, set

$$
L(f, g)=\sum_{l=0}^{n}(-1)^{l} \operatorname{Tr}\left(\Phi_{l}^{-1} g_{*} \Phi_{l} f^{*}\right),
$$

where $\Phi_{i}: H^{i}(M ; \mathbf{Q}) \rightarrow H_{n-i}(M ; \mathbf{Q})$ is the Poincaré duality isomorphism. If $L(f, g) \neq 0$, then $f$ and $g$ have a coincidence.

REMARKS. 1. It is immediate that $L(f, \mathrm{id})=L(f)$, the ordinary Lefschetz number of $f$, so this result implies the Lefschetz fixed-point theorem for $M$.

2. It follows from properties of trace that $L(f, g)=(-1)^{n} L(g, f)$.

Let $g$ be a self-map of $M$. We define the degree of $g$ by $g_{*}[M]=$ $(\operatorname{deg} g)[M]$, where $[M] \in H_{n}(M ; \mathbf{Q})$ is the fundamental class of $M$. The following result is useful in computing the Lefschetz coincidence number.

Proposition 3.2. If $g$ is a self-map of $M$ with $\operatorname{deg} g \neq 0$, then $g^{*}$ : $H^{*}(M ; \mathbf{Q}) \rightarrow H^{*}(M ; \mathbf{Q})$ has an inverse $\bar{g}^{*}$ and

$$
L(f, g)=(\operatorname{deg} g) L\left(\bar{g}^{*} f^{*}\right)
$$

for any other self-map $f$ of $M$.

Proof. If $\operatorname{deg} g \neq 0$, it follows from consideration of Poincaré duality that $g^{*}$ is injective. Then $g^{*}$ is an automorphism, since each vector space $H^{i}(M ; \mathbf{Q})$ is finite-dimensional. For $u \in H^{i}(M ; \mathbf{Q})$,

$$
\begin{aligned}
\Phi_{1}^{-1} g_{*} \Phi_{l} f^{*}(u) & =\Phi_{i}^{-1} g_{*}\left(g^{*} \bar{g}^{*} f^{*}(u) \cap[M]\right) \\
& =\Phi_{l}^{-1}\left(\bar{g}^{*} f^{*}(u) \cap g_{*}[M]\right)=(\operatorname{deg} g) \bar{g}^{*} f^{*}(u),
\end{aligned}
$$

and the conclusion follows from the definition of $L(f, g)$.

By Theorem 3.1, any fixed-point-free self-map $f$ of $M$ must have $L(f)=0$, and any pair $f, g$ of self-maps without a coincidence must have $L(f, g)=0$. We put

$$
L Z(M)=\left\{f^{*} \mid f: M \rightarrow M \text { and } L(f)=0\right\}
$$


and say $f^{*}, g^{*} \in L Z(M)$ are compatible if $L(f, g)=0$. If $\chi(M) \neq 0$ and $L Z(M)$ consists of automorphisms of $H^{*}(M ; \mathbf{Q})$, we call $M L$-rigid. We then have the following result.

Proposition 3.3. Suppose $M$ is L-rigid. If $\# M \geq k+1$, then $L Z(M)$ contains a subset of $k$ pairwise compatible elements.

Proof. By the hypothesis, there is a set $S$ of $k$ pairwise noncoincident fixed-point-free self-maps of $M$. Let $f, g \in S$. Then $f^{*}$ and $g^{*}$ are compatible elements of $L Z(M)$. We have $f^{*} \neq g^{*}$, since otherwise

$$
L(f, g)=L(f, f)=(\operatorname{deg} f) L(\mathrm{id})=(\operatorname{deg} f) \chi(M) \neq 0 .
$$

Thus, $\left\{f^{*} \mid f \in S\right\}$ is a set of $k$ pairwise compatible elements of $L Z(M)$.

REMARK. Note that if $M$ is $L$-rigid, then any pair $f^{*}, g^{*} \in L Z(M)$ is compatible if and only if $L\left(\bar{g}^{*} f^{*}\right)=0$.

It follows immediately from 3.3 that

$$
\# M \leq \operatorname{card} L Z(M)+1
$$

when $M$ is $L$-rigid and $L Z(M)$ is finite. Thus we have, e.g., $\# S^{2 n} \leq 2$ for any even sphere $S^{2 n}$ (and in fact $\# S^{2 n}=2$, since the antipodal map is fixed-point-free). As we see in the next section, however, 3.3 sometimes gives a sharper upper bound than (1).

4. Noncoincidence index of some flag manifolds. Let $F\left(1^{k}, n\right)$ denote the homogeneous space $U(n+k) / H$, where $H$ is conjugate to $U(1)^{k} \times U(n)$. (We can assume $n=0$ or $n \geq 2$ : in the former case we write $F\left(1^{k}\right)$ instead of $F\left(1^{k}, 0\right)$.) It is proved in [7] that $\# F\left(1^{k}\right)=k$ !. In this section we compute $\# F\left(1^{k}, n\right)$ for all $k$ and $n$.

The manifold $F\left(1^{k}, n\right)$ can be thought of as in the space of $k$-tuples of orthogonal lines in $\mathbf{C}^{n+k}$. Thus, there are maps

$$
\pi_{i}: F\left(1^{k}, n\right) \rightarrow \mathrm{C} P^{n+k-1}, \quad 1 \leq i \leq k,
$$

given by picking out the $i$ th line. If we let $t \in H^{2}\left(\mathbf{C} P^{n+k-1} ; \mathbf{Q}\right)$ be the first Chern class of the canonical line bundle over $\mathbf{C} P^{n+k-1}$ and put $t_{i}=\pi_{i}^{*}(t)$, we have the following description of $H^{*}\left(F\left(1^{k}, n\right) ; \mathbf{Q}\right)[\mathbf{1}]$.

$$
H^{*}\left(F\left(1^{k}, n\right) ; \mathbf{Q}\right)=\mathbf{Q}\left[t_{1}, t_{2}, \ldots, t_{k}\right] /\left\{h_{i} \mid n+1 \leq i \leq n+k\right\},
$$

where $h_{i}$ is the $i$ th complete symmetric function in $t_{1}, t_{2}, \ldots, t_{k}$, i.e.

$$
h_{i}=\sum_{p_{1}+\cdots+p_{k}=i} t_{1}^{p_{1}} t_{2}^{p_{2}} \cdots t_{k}^{p_{k}}
$$


There is a free action of the symmetric group $\Sigma_{k}$ on $F\left(1^{k}, n\right)$ by permutation of lines, and this action evidently permutes the $t_{i}$ in cohomology.

For any $m \in \mathbf{Q}$ and $\sigma \in \Sigma_{k}$, let $h_{m}^{\sigma}$ denote the endomorphism of $H^{*}\left(F\left(1^{k}, n\right) ; \mathbf{Q}\right)$ given by

$$
h_{m}^{\sigma}\left(t_{i}\right)=m t_{\sigma(i)}
$$

The following classification theorem for endomorphisms of $H^{*}\left(F\left(1^{k}, n\right)\right.$; Q) is proved in $\S 5$.

THEOREM 4.1. Unless $k=2$ and $n$ is a positive even number, all endomorphisms of $H^{*}\left(F\left(1^{k}, n\right) ; \mathbf{Q}\right)$ are of the form $h_{m}^{\sigma}$ for some $m \in \mathbf{Q}$ and $\sigma \in \Sigma_{k}$. If $k=2$ and $n \geq 2$ is even, the only additional endomorphisms are

$$
t_{i} \rightarrow(-1)^{i} m t_{q}, \quad i=1,2,
$$

for $q \in\{1,2\}$ and $m \in \mathbf{Q}$.

The next result gives a formula for $L\left(h_{m}^{\sigma}\right)$.

THEOREM 4.2. Let $\lambda_{1} \geq \lambda_{2} \geq \cdots$ be the cycle-type of $\sigma \in \Sigma_{k}$ (so $\lambda_{1}$ is the length of the longest cycle in $\sigma, \lambda_{2}$ is the length of the next longest cycle, etc.). Then $h_{m}^{\sigma}: H^{*}\left(F\left(1^{k}, n\right) ; \mathbf{Q}\right) \rightarrow H^{*}\left(F\left(1^{k}, n\right) ; \mathbf{Q}\right)$ has Lefschetz number

$$
L\left(h_{m}^{\sigma}\right)=\frac{\left(1-m^{n+1}\right)\left(1-m^{n+2}\right) \cdots\left(1-m^{n+k}\right)}{\left(1-m^{\lambda_{1}}\right)\left(1-m^{\lambda_{2}}\right) \cdots} .
$$

Proof. For $h_{m}^{\sigma}: H^{*}\left(F\left(1^{k}, n\right) ; \mathbf{Q}\right) \rightarrow H^{*}\left(F\left(1^{k}, n\right)\right.$; Q), let $P_{k, n}(\sigma, m)$ denote $L\left(h_{m}^{\sigma}\right)$. From [7] we have the formula

$$
P_{k, 0}(\sigma, m)=\frac{(1-m)\left(1-m^{2}\right) \cdots\left(1-m^{k}\right)}{\left(1-m^{\lambda_{1}}\right)\left(1-m^{\lambda_{2}}\right) \cdots} .
$$

Now the spectral sequence of the fibration

$$
F\left(1^{k}\right) \rightarrow F\left(1^{k}, n\right) \rightarrow G_{k}\left(\mathbf{C}^{n+k}\right),
$$

where $G_{k}\left(\mathbf{C}^{n+k}\right)$ is the Grassmannian of $k$-planes in $\mathbf{C}^{n+k}$, collapses for degree reasons. Thus

$$
H^{*}\left(F\left(1^{k}, n\right) ; \mathbf{Q}\right) \cong H^{*}\left(F\left(1^{k}\right) ; \mathbf{Q}\right) \otimes H^{*}\left(G_{k}\left(\mathbf{C}^{n+k}\right) ; \mathbf{Q}\right)
$$

additively. Now $H^{*}\left(G_{k}\left(\mathbf{C}^{n+k}\right)\right.$; $\left.\mathbf{Q}\right)$ can be regarded as the invariant subring of $H^{*}\left(F\left(1^{k}, n\right)\right.$; $\left.\mathbf{Q}\right)$ under the $\Sigma_{k^{-a c t i o n}}$, and $H^{*}\left(F\left(1^{k}\right)\right.$; $\left.\mathbf{Q}\right)$ is a quotient of $H^{*}\left(F\left(1^{k}, n\right)\right.$; Q) (the projection is the obvious map sending $t_{l} \in H^{2}\left(F\left(1^{k}, n\right) ; \mathbf{Q}\right)$ to $\left.t_{l} \in H^{2}\left(F\left(1^{k}\right) ; \mathbf{Q}\right)\right)$. Any endomorphism $h_{m}^{\sigma}$ of 
$H^{*}\left(F\left(1^{k}, n\right) ; \mathbf{Q}\right)$ restricts to the endomorphism of $H^{*}\left(G_{k}\left(\mathbf{C}^{n+k}\right)\right.$; Q $)$ which multiplies dimension $2 i$ by $m^{i}$, and gives rise to the corresponding $h_{m}^{\sigma}$ on $H^{*}\left(F\left(1^{k}\right) ; \mathbf{Q}\right)$. Since trace is multiplicative on tensor products,

$$
P_{k, n}(\sigma, m)=P_{k, 0}(\sigma, m) \sum_{i \geq 0} m^{i} \operatorname{dim} H^{2 i}\left(G_{k}\left(\mathbf{C}^{n+k}\right) ; \mathbf{Q}\right) \text {. }
$$

It is well known (see e.g. [1]) that

$\sum_{i \geq 0} m^{i} \operatorname{dim} H^{2 i}\left(G_{k}\left(\mathbf{C}^{n+k}\right) ; \mathbf{Q}\right)=\frac{\left(1-m^{n+1}\right)\left(1-m^{n+2}\right) \cdots\left(1-m^{n+k}\right)}{(1-m)\left(1-m^{2}\right) \cdots\left(1-m^{k}\right)}$, and this together with (2) and (3) implies the conclusion.

We can use Theorems 4.1 and 4.2 to show that many of the manifolds $F\left(1^{k}, n\right)$ are $L$-rigid.

Proposition 4.3. Suppose $k \neq 2$, $n$ is odd, or $n=0$. Then $F\left(1^{k}, n\right)$ is $L$-rigid. Further, if $\Gamma$ is the set of products of $[k / 2]$ disjoint transpositions in $\Sigma_{k}$, then

$$
L Z\left(F\left(1^{k}, n\right)\right)= \begin{cases}\left\{h_{1}^{\sigma} \mid \sigma \neq \mathrm{id}\right\} \cup\left\{h_{-1}^{\sigma} \mid \sigma \in \Sigma_{k}\right\}, & \text { kn odd }, \\ \left\{h_{1}^{\sigma} \mid \sigma \neq \mathrm{id}\right\} \cup\left\{h_{-1}^{\sigma} \mid \sigma \notin \Gamma\right\}, & \text { kn even. }\end{cases}
$$

Proof. By 4.1, an element of $\operatorname{LZ}\left(F\left(1^{k}, n\right)\right)$ must be an endomorphism $h_{m}^{\sigma}$ of $H^{*}\left(F\left(1^{k}, n\right)\right.$; Q) with $L\left(h_{m}^{\sigma}\right)=0$. The above list follows from consideration of (1) (it is easy to see that any $h_{m}^{\sigma}, m= \pm 1$, is induced by a self-map of $\left.F\left(1^{k}, n\right)\right)$. Now

$$
\chi\left(F\left(1^{k}, n\right)\right)=k !\left(\begin{array}{c}
n+k \\
k
\end{array}\right)
$$

and clearly $h_{m}^{\sigma}$ is an automorphism for $m \neq 0$ (in fact $\operatorname{deg} h_{m}^{\sigma}=m^{d} \operatorname{sgn} \sigma$, where $d=\operatorname{dim}_{\mathrm{C}} F\left(1^{k}, n\right)$ ), so $F\left(1^{k}, n\right)$ is $L$-rigid.

Now we can give an upper bound for $\# F\left(1^{k}, n\right)$ when $n$ and $k$ satisfy the hypothesis of the preceding result.

Proposition 4.4. Suppose $k \neq 2, n$ is odd, or $n=0$. Then $\# F\left(1^{k}, n\right)$ $\leq k$ ! if $k n$ is even, and $\# F\left(1^{k}, n\right) \leq 2 k$ ! if $k n$ is odd.

Proof. The statement about \#F( $\left.1^{k}, n\right)$ for $k n$ odd follows immediately from 3.3 , since $\operatorname{LZ}\left(F\left(1^{k}, n\right)\right)$ has $2 k !-1$ elements by 4.3 . Again by 3.3 , to prove the statement about $\# F\left(1^{k}, n\right)$ for $k n$ even it suffices to show that any set of pairwise compatible elements of $\operatorname{LZ}\left(F\left(1^{k}, n\right)\right)$ has at most $k !-1$ elements in this case. Suppose $k n$ even and let $S \subset \operatorname{LZ}\left(F\left(1^{k}, n\right)\right)$ be a set of compatible elements. Let

$$
\begin{aligned}
& H=\left\{\sigma \in \Sigma_{k} \mid h_{1}^{\sigma} \in S\right\} \cup\{\text { id }\}, \\
& K=\left\{\sigma \in \Sigma_{k} \mid h_{-1}^{\sigma} \in S\right\} .
\end{aligned}
$$


Then $\operatorname{card} S=\operatorname{card} H+\operatorname{card} K-1$. Since

$$
L\left(h_{-1}^{\tau}, h_{1}^{\sigma}\right)=\left(\operatorname{deg} h_{1}^{\sigma}\right) L\left(h_{1}^{\sigma^{-1}} h_{-1}^{\tau}\right)= \pm L\left(h_{-1}^{\sigma^{-1} \tau}\right),
$$

we must have $\sigma^{-1} \tau \notin \Gamma$ for $\tau \in K$ and $\sigma \in H$ (here $\Gamma$ is as in 4.3). But then, if we take $\rho \in \Gamma$, we have $\sigma \rho \notin K$ for every $\sigma \in H$ : hence card $H+$ card $K \leq k$ !, and the conclusion follows.

Next we show that the inequalities of 4.4 are equalities. To do this, we construct fixed-point-free, noncoincident maps. For any even number $2 r$, define $J: \mathbf{C}^{2 r} \rightarrow \mathbf{C}^{2 r}$ by

$$
J\left(z_{1}, z_{2}, \ldots, z_{2 r-1}, z_{2 r}\right)=\left(-\bar{z}_{2}, \bar{z}_{1}, \ldots,-\bar{z}_{2 r}, \bar{z}_{2 r-1}\right) .
$$

Then $J$ is a conjugate-linear map of $\mathbf{C}^{2 r}$ with $J^{2}=-$ id. Under the evident identification $\mathbf{C}^{2 r} \cong \mathbf{H}^{r}$, we can regard $J$ as multiplication by the quaternion $j$. Any subspace of $\mathbf{C}^{2 r}$ invariant under $J$ can be given the structure of a quaternionic vector space, and thus must be even-dimensional (cf. the proof of Theorem 1 of [6]). Further, if $\langle$,$\rangle denotes inner product,$

$$
\langle J v, J w\rangle=\langle w, v\rangle \text { for } v, w \in \mathbf{C}^{2 r} .
$$

Thus $J$ preserves orthogonality.

THEOREM 4.5. Unless $k=2$ and $n$ is a positive even number,

$$
\# F\left(1^{k}, n\right)= \begin{cases}2 k !, & k n \text { odd }, \\ k !, & k n \text { even } .\end{cases}
$$

Proof. Since there is a free $\Sigma_{\mathrm{k}}$-action on $F\left(1^{k}, n\right)$ (i.e., permutation of lines), we have $\# F\left(1^{k}, n\right) \geq k$ !; together with 4.4 , this disposes of the case $k n$ even. Now suppose $k n$ is odd. Then $n+k$ is even, and we have the map $J: \mathbf{C}^{n+k} \rightarrow \mathbf{C}^{n+k}$ defined above. Consider the $2 k !-1$ self-maps of $F\left(1^{k}, n\right)$ defined by

$$
\left(l_{1}, l_{2}, \ldots, l_{k}\right) \rightarrow\left(l_{\pi(1)}, l_{\pi(2)}, \ldots, l_{\pi(k)}\right), \quad \pi \in \Sigma_{k}-\{\mathrm{id}\}
$$

and

$$
\left(l_{1}, l_{2}, \ldots, l_{k}\right) \rightarrow\left(J l_{\pi(1)}, J l_{\pi(2)}, \ldots, J l_{\pi(k)}\right), \quad \pi \in \Sigma_{k} .
$$

We claim these maps are fixed-point-free and pairwise noncoincident. Clearly the maps in (5) are fixed-point-free and pairwise noncoincident, and the maps in (6) are pairwise noncoincident. Suppose now we have a fixed point of a map in (6) or a coincidence between a map in (5) and one in (6), i.e., an element $\left(l_{1}, l_{2}, \ldots, l_{k}\right)$ of $F\left(1^{k}, n\right)$ with

$$
l_{\pi(i)}=J l_{\sigma(l)}, \quad 1 \leq i \leq k,
$$


for some $\pi, \sigma \in \Sigma_{k}$. Then $J$ fixes $l_{1} \oplus l_{2} \oplus \cdots \oplus l_{k}$. But this is impossible, since $J$ cannot fix an odd-dimensional subspace of $\mathbf{C}^{n+k}$.

Finally, we dispose of the case $k=2$ and $n \geq 2$ even.

THEOREM 4.6. If $n \geq 2$ is even, then $\# F\left(1^{2}, n\right)=\infty$.

Proof. Let $J: \mathbf{C}^{n+2} \rightarrow \mathbf{C}^{n+2}$ be as defined above. Note that for $v \in \mathbf{C}^{n+2}$,

$$
\langle J v, v\rangle=\left\langle J v, J^{2} v\right\rangle=-\langle J v, v\rangle
$$

by (4) above; thus $\langle J v, v\rangle=0$, and $J l$ is orthogonal to $l$ for any line $l$. Define $\psi: F\left(1^{2}, n\right) \rightarrow F\left(1^{2}, n\right)$ by $\psi\left(l_{1}, l_{2}\right)=\left(J l_{1}, l_{1}\right):$ then $\psi$ is fixedpoint-free and nonsurjective, and the conclusion follows by 2.2.

5. Proof of the endomorphism theorem. This section is devoted to a proof of Theorem 4.1. We use the notation of the previous section.

Since $t_{i} \in H^{2}\left(F\left(1^{k}, n\right) ; \mathbf{Q}\right)$ is pulled back from $H^{2}\left(\mathbf{C} P^{n+k-1} ; \mathbf{Q}\right)$, we have $t_{i}^{n+k}=0$. The next result gives a converse: it is proved in [8] for $k \leq n$, and in [2] without restriction.

TheOREM 5.1. If $u \in H^{2}\left(F\left(1^{k}, n\right)\right.$; $\left.\mathbf{Q}\right)$ and $u^{n+k}=0$, then $u$ is of the form at ${ }_{i}$ for some $a \in \mathbf{Q}$ and $1 \leq i \leq k$.

Now suppose $f$ is an endomorphism of $H^{*}\left(F\left(1^{k}, n\right) ; \mathbf{Q}\right)$. Then $f\left(t_{i}\right)^{n+k}=f\left(t_{i}^{n+k}\right)=0$ for $1 \leq i \leq k$, and it follows from 5.1 that

$$
f\left(t_{i}\right)=m_{i} t_{p(l)}, \quad 1 \leq i \leq k,
$$

for some function $p:\{1,2, \ldots, k\} \rightarrow\{1,2, \ldots, k\}$ and rational numbers $m_{l}$. We shall prove that $p$ is a permutation and all the $m_{i}$ are equal unless $k=2$ and $n$ is even.

First we prove a technical lemma. The expression $h_{l}\left(x_{1}, x_{2}, \ldots, x_{r}\right)$ denotes the $i$ th complete symmetric function in $x_{1}, x_{2}, \ldots, x_{r}$.

LEMMA 5.2. Let $n \geq 0$ be an integer, $a_{1}, a_{2}, \ldots, a_{r}$ real numbers, and suppose

$$
h_{n+i}\left(a_{1}, a_{2}, \ldots, a_{r}\right)=0, \quad 1 \leq i \leq r-1 .
$$

Then unless $r=2$ and $n$ is even, $a_{1}=a_{2}=\cdots=a_{r}=0$. If $r=2$ and $n$ is even, $a_{2}=-a_{1}$.

Proof. Suppose first that $r=2$. Then we have

$$
h_{n+1}\left(a_{1}, a_{2}\right)=a_{1}^{n+1}+a_{1}^{n} a_{2}+\cdots+a_{1} a_{2}^{n}+a_{2}^{n+1}=0 .
$$


If $a_{1}=a_{2}$, this evidently implies $a_{1}=a_{2}=0$. If $a_{1} \neq a_{2}$, then (1) is

$$
\frac{a_{1}^{n+2}-a_{2}^{n+2}}{a_{1}-a_{2}}=0
$$

from which it follows that $a_{2}=-a_{1}$ and $n$ is even.

Now suppose $r=3$. We have

$$
h_{n+1}\left(a_{1}, a_{2}, a_{3}\right)=h_{n+2}\left(a_{1}, a_{2}, a_{3}\right)=0 .
$$

Then from the relations

$$
\begin{aligned}
h_{n+2}\left(a_{1}, a_{2}, a_{3}\right)= & a_{1}^{n+2}+a_{1}^{n+1} h_{1}\left(a_{2}, a_{3}\right)+\cdots+a_{1} h_{n+1}\left(a_{2}, a_{3}\right) \\
& +h_{n+2}\left(a_{2}, a_{3}\right)
\end{aligned}
$$

and

$$
a_{1} h_{n+1}\left(a_{1}, a_{2}, a_{3}\right)=a_{1}^{n+2}+a_{1}^{n+1} h_{1}\left(a_{2}, a_{3}\right)+\cdots+a_{1} h_{n+1}\left(a_{2}, a_{3}\right)
$$

we get

$$
h_{n+2}\left(a_{2}, a_{3}\right)=0 \text {. }
$$

Similarly,

$$
h_{n+2}\left(a_{1}, a_{3}\right)=0
$$

and

$$
h_{n+2}\left(a_{1}, a_{2}\right)=0 .
$$

By the argument of the preceding paragraph, these equations imply $a_{1}=a_{2}=a_{3}=0$ unless $n$ is odd. In this case, (2) gives $a_{3}=-a_{2}$, (4) gives $a_{2}=-a_{1}$, and (3) gives $a_{1}=-a_{3}$ : but then $a_{1}=a_{2}=a_{3}=0$. It is now clear how to prove the result by induction for any $r>3$.

As noted in the previous section, $H^{*}\left(F\left(1^{k}, n\right)\right.$; $\left.\mathbf{Q}\right)$ is the quotient of $\mathbf{Q}\left[t_{1}, \ldots, t_{k}\right]$ by the ideal generated by $R_{1}, R_{2}, \ldots, R_{k}$, where

$$
R_{l}=h_{n+l}\left(t_{1}, t_{2}, \ldots, t_{k}\right), \quad 1 \leq i \leq k .
$$

For $f$ to be a well-defined endomorphism of $H^{*}\left(F\left(1^{k}, n\right)\right.$; $\left.\mathbf{Q}\right)$ there must be relations

$$
f\left(R_{i}\right)=N_{i} R_{i}+\sum_{1 \leq|\alpha|<i} N_{l}^{\alpha} t^{\alpha} R_{l-|\alpha|}, \quad 1 \leq i \leq k,
$$

in $\mathbf{Q}\left[t_{1}, \ldots, t_{k}\right]$, where the sum is over multi-indices $\alpha=\left(\alpha_{1}, \alpha_{2}, \ldots, \alpha_{k}\right)$ with $|\alpha|=\alpha_{1}+\alpha_{2}+\cdots+\alpha_{k}$ and

$$
t^{\alpha}=t_{1}^{\alpha_{1}} t_{2}^{\alpha_{2}} \cdots t_{k}^{\alpha_{k}} .
$$

Then we have the following result. 
LEMMA 5.3. If $r$ of the elements $t_{1}, t_{2}, \ldots, t_{k}$ are missing from the image of $f$, then $f\left(R_{i}\right)=0$ in $\mathbf{Q}\left[t_{1}, \ldots, t_{k}\right]$ for $1 \leq i \leq r$.

Proof. Permuting the $t_{i}$ if necessary, we can assume that $t_{1}, t_{2}, \ldots, t_{r}$ are missing from the image of $f$. Define $\pi: F\left(1^{k}, n\right) \rightarrow F\left(1^{k-r}, n+r\right)$ by

$$
\pi\left(l_{1}, l_{2}, \ldots, l_{k}\right)=\left(l_{r+1}, \ldots, l_{k}\right) .
$$

Then $\pi^{*}$ sends $t_{\imath} \in H^{2}\left(F\left(1^{k-r}, n+r\right)\right.$; $\left.\mathbf{Q}\right)$ to $t_{r+i} \in H^{2}\left(F\left(1^{k}, n\right)\right.$; $\left.\mathbf{Q}\right)$ for $1 \leq i \leq k-r$, and is injective since the spectral sequence of the fibration

$$
F\left(1^{r}, n\right) \rightarrow F\left(1^{k}, n\right) \stackrel{\pi}{\rightarrow} F\left(1^{k-r}, n+r\right)
$$

collapses for degree reasons. Now $t_{1}, \ldots, t_{r}$ are missing from $\operatorname{im} f$, so $\operatorname{im} f \subset \operatorname{im} \pi^{*}$ in $H^{*}\left(F\left(1^{k}, n\right)\right.$; Q). Hence $f=\pi^{*} g$ for

$$
g=\left(\pi^{*}\right)^{-1} f: H^{*}\left(F\left(1^{k}, n\right) ; \mathbf{Q}\right) \rightarrow H^{*}\left(F\left(1^{k-r}, n+r\right) ; \mathbf{Q}\right) .
$$

But the first nontrivial relation in $H^{*}\left(F\left(1^{k-r}, n+r\right)\right.$; Q $)$ is in dimension $2(n+r+1)$, so $g\left(R_{l}\right)=0$ in $\mathbf{Q}\left[t_{1}, \ldots, t_{k-r}\right]$ for $1 \leq i \leq r$ and the conclusion follows.

Suppose $n \geq 2$. For each $i$ from 1 to $k$, we define the weight of $i$ to be the cardinality of $\left\{r \mid p(r)=i\right.$ and $\left.m_{r} \neq 0\right\}$, i.e., the number of $t_{r}$ that $f$ maps to $t_{l}$ with nonzero coefficient. The following result is the key to the proof of Theorem 4.1 .

Proposition 5.4. Let $n \geq 2$. Then $i$ has weight at most 1 for $1 \leq i \leq k$ unless $k=2$ and $n$ is even. If $k=2, n$ is even, and $q \in\{1,2\}$ has weight 2 , then $f$ has the form

$$
f\left(t_{i}\right)=(-1)^{i} m t_{q}, \quad i=1,2 .
$$

Proof. Suppose $q$ has weight $w>1$. Then at least $w-1$ of the $t_{l}$ are missing from the image of $f$, and by 5.3

$$
f\left(R_{i}\right)=0, \quad 1 \leq i \leq w-1,
$$

in $\mathrm{Q}\left[t_{1}, \ldots, t_{k}\right]$. We can assume $t_{1}, t_{2}, \ldots, t_{w}$ map to $t_{q}$ with nonzero coefficient. Examine the coefficient of $t_{q}^{n+\imath}$ in (6) to get

$$
h_{l}\left(m_{1}, m_{2}, \ldots, m_{w}\right)=0, \quad 1 \leq i \leq w-1 .
$$

Then unless $w=2$ and $n$ is even, $m_{1}=m_{2}=\cdots=m_{w}=0$ by 5.2, a contradiction. If $w=2$ and $n$ is even, 5.2 gives $m_{2}=-m_{1} \neq 0$. In this case, $f\left(R_{1}\right)=0$ and $f\left(R_{2}\right) \neq 0$ in $\mathbf{Q}\left[t_{1}, \ldots, t_{k}\right]$ : but then no $i$ can have weight 1 and no more than one $t_{i}$ can be missing from the image of $f$, from which follows $k=2$. 
REMARK. The case $n=0$ is disposed of in [7], where it is proved that all endomorphisms of $H^{*}\left(F\left(1^{k}\right) ; \mathbf{Q}\right)$ have the form $h_{m}^{\sigma}$.

By the preceding result, the function $p$ of $\{1,2, \ldots, k\}$ can be assumed a permutation if $k \neq 2$ or $n$ is odd. To finish the proof of 4.1, we need only show all the $m_{i}$ are equal in this case. Now in $f\left(R_{1}\right)$ the coefficient of $t_{r}^{n+1}$ is $m_{s}^{n+1}$, where $p(s)=r$. The coefficient of $t_{r}^{n+1}$ on the right-hand side of (5) (with $i=1$ ) is $N_{1}$. Thus $m_{s}^{n+1}=N_{1}$ for $1 \leq s \leq k$. For $n$ even, this shows all the $m_{s}$ are equal. For $n$ odd, it is also necessary to inspect the coefficients of terms $t_{r}^{n} t_{s}, r \neq s$, in equation (5) with $i=1$.

\section{REFERENCES}

[1] A. Borel, Sur la cohomologie des espaces fibrés principaux et des espaces homogènes de groupes de Lie compacts, Ann. of Math., 57 (1953), 115-207.

[2] S. A. Broughton, M. Hoffman and W. Homer, The height of two-dimensional cohomology classes of complex flag manifolds, Canad. Math. Bull., 26 (1983), 498-502.

[3] R. F. Brown, Path fields on manifolds, Trans. Amer. Math. Soc., 118 (1965), 180-191.

[4] R. F. Brown and E. Fadell, Nonsingular path fields on compact topological manifolds, Proc. Amer. Math. Soc., 16 (1965), 1342-1349.

[5] E. Fadell and L. Neuwirth, Configuration spaces, Math. Scand., 10 (1962), 111-118.

[6] H. Glover and W. Homer, Fixed points on flag manifolds, Pacific J. Math., 101 (1982), 303-306.

[7] M. Hoffman, On fixed point free maps of the complex flag manifold, Indiana U. Math. J., 33 (1984), 249-255.

[8] A. Liulevicius, Homotopy rigidity of linear actions: characters tell all, Bull. Amer. Math. Soc., 84 (1978), 213-221.

[9] J. Vick, Homology Theory, Academic Press, New York, 1973.

Received March 4, 1983 and in revised form April 29, 1983.

OHIO STATE UNIVERSITY

Columbus, OH 43210

Current address: U. S. Naval Academy Annapolis, MD 21402 



\title{
PACIFIC JOURNAL OF MATHEMATICS EDITORS
}

\author{
Donald BABBITt (Managing Editor) \\ University of California \\ Los Angeles, CA 90024 \\ J. DugunduI \\ University of Southern California \\ Los Angeles, CA 90089-1113 \\ R. FINN \\ Stanford University \\ Stanford, CA 94305 \\ HermanN FlaschKa \\ University of Arizona \\ Tucson, AŻ 85721
}

C. C. Moore

University of California

Berkeley, CA 94720

ARTHUR Ogus

University of California

Berkeley, CA 94720

Hugo Rossi

University of Utah

Salt Lake City, UT 84112

H. SAMELSON

Stanford University

Stanford, CA 94305

ASSOCIATE EDITORS
R. ARENS
E. F. BECKENBACH
B. H. NEUMANN
F. WOLF
K. YoSHIDA (1906-1982)

\section{SUPPORTING INSTITUTIONS}

$\begin{array}{ll}\text { UNIVERSITY OF ARIZONA } & \text { UNIVERSITY OF OREGON } \\ \text { UNIVERSITY OF BRITISH COLUMBIA } & \text { UNIVERSITY OF SOUTHERN CALIFORNIA } \\ \text { CALIFORNIA INSTITUTE OF TECHNOLOGY } & \text { STANFORD UNIVERSITY } \\ \text { UNIVERSITY OF CALIFORNIA } & \text { UNIVERSITY OF HAWAII } \\ \text { MONTANA STATE UNIVERSITY } & \text { UNIVERSITY OF TOKYO } \\ \text { UNIVERSITY OF NEVADA, RENO } & \text { UNIVERSITY OF UTAH } \\ \text { NEW MEXICO STATE UNIVERSITY } & \text { WASHINGTON STATE UNIVERSITY } \\ \text { OREGON STATE UNIVERSITY } & \text { UNIVERSITY OF WASHINGTON }\end{array}$

The Supporting Institutions listed above contribute to the cost of publication of this Journal, but they are not owners or publishers and have no responsibility for its content or policies.

Mathematical papers intended for publication in the Pacific Journal of Mathematics should be in typed form or offset-reproduced (not dittoed), double spaced with large margins. Please do not use built up fractions in the text of the manuscript. However, you may use them in the displayed equations. Underline Greek letters in red, German in green, and script in blue. The first paragraph must be capable of being used separately as a synopsis of the entire paper. In particular it should contain no bibliographic references. Please propose a heading for the odd numbered pages of less than 35 characters. Manuscripts, in triplicate, may be sent to any one of the editors. Please classify according to the scheme of Math. Reviews, Index to Vol. 39. Supply name and address of author to whom proofs should be sent. All other communications should be addressed to the managing editor, or Elaine Barth, University of California, Los Angeles, California 90024.

There are page-charges associated with articles appearing in the Pacific Journal of Mathematics. These charges are expected to be paid by the author's University, Government Agency or Company. If the author or authors do not have access to such Institutional support these charges are waived. Single authors will receive 50 free reprints; joint authors will receive a total of 100 free reprints. Additional copies may be obtained at cost in multiples of 50 .

The Pacific Journal of Mathematics is issued monthly as of January 1966. Regular subscription rate: $\$ 190.00$ a year (5 Vols., 10 issues). Special rate: $\$ 66.00$ a year to individual members of supporting institutions.

Subscriptions, orders for numbers issued in the last three calendar years, and changes of address should be sent to Pacific Journal of Mathematics, P.O. Box 969, Carmel Valley, CA 93924, U.S.A. Old back numbers obtainable from Kraus Periodicals Co., Route 100, Millwood, NY 10546.

The Pacific Journal of Mathematics at P.O. Box 969, Carmel Valley, CA 93924 (ISSN 0030-8730) publishes 5 volumes per year. Application to mail at Second-class postage rates is pending at Carmel Valley, California, and additional mailing offices. Postmaster: Send address changes to Pacific Journal of Mathematics, P.O. Box 969, Carmel Valley, CA 93924.

PUBLISHED BY PACIFIC JOURNAL OF MATHEMATICS, A NON-PROFIT CORPORATION

Copyright $@ 1984$ by Pacific Journal of Mathematics 


\section{Pacific Journal of Mathematics}

\section{Vol. 115, No. $2 \quad$ October, 1984}

Ersan Akyildiz, Gysin homomorphism and Schubert calculus ...........257

Marilyn Breen, Clear visibility and unions of two starshaped sets in the

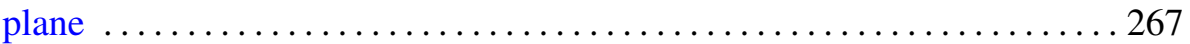

Robert F. Brown, Retraction methods in Nielsen fixed point theory . ......277

Herbert Busemann and Bhalchandra B. Phadke, A general version of Beltrami's theorem in the large ............................... 299

Gerald Arthur Edgar and Robert Francis Wheeler, Topological properties of Banach spaces ............................... 317

Yaakov Friedman and Bernard Russo, Conditional expectation without

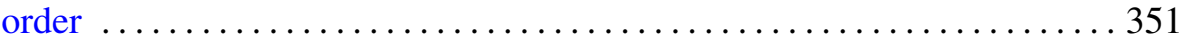

Robert Allen Goggins, Cobordism of manifolds with strong almost tangent

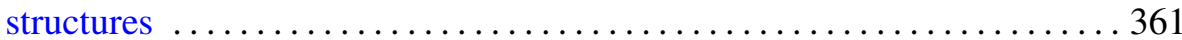

Mike Hoffman, Noncoincidence index of manifolds . . . . . . . . . . . . . 373

William H. Julian, $\varepsilon$-continuity and monotone operations $\ldots \ldots \ldots \ldots 385$

Gerasimos E. Ladas, Y. G. Sficas and I. P. Stavroulakis, Nonoscillatory

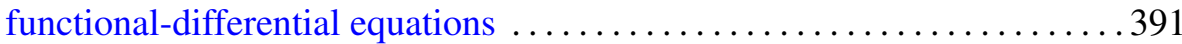

Arnold William Miller and Karel Libor Prikry, When the continuum has

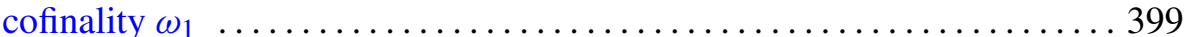

Jean-Leah Mohrherr, Density of a final segment of the truth-table

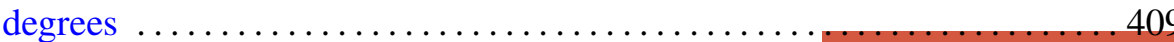

Carl Norman Mutchler, The flat Cauchy problem for radially hyperbolic operators from a characteristic manifold of high codimension ...

Kenji Nakagawa, On the orders of automorphisms of a closed Riemann surface

W. Ricker, Representation of vector-valued functions by Laplace transforms

Jorge Donato Samur, On semigroups of convolution operators in Hilbert

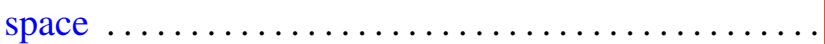

Joseph Gail Stampfli, One-dimensional perturbations of operators 481 Andrew George Earnest and John Sollion Hsia, Correction to: "Spinor norms of local integral rotations. II" 